\title{
TRAVELING-WAVE ACCELERATING TEST STRUCTURE AT 34.3 GHZ
}

\author{
O. A. Nezhevenko and V. P. Yakovlev \\ Omega-P Inc., 345 Whitney Avenue, New Haven, CT 06511
}

\begin{abstract}
A future electron-positron linear collider with c.m. energy in the range of $3-5 \mathrm{TeV}$ is understood to require an operating RF frequency in the range of 30-100 GHz. Higher operating frequency can lead to higher acceleration gradient, with a corresponding smaller accelerator length. This paper describes a test structure designed to determine the maximum achievable accelerating gradient for an operating frequency of 34.272 $\mathrm{GHz}$. The $\sim 6-\mathrm{cm}$ long test structure consists of 21 cells operating in the $2 \pi / 3$ mode. It is to be driven using a magnicon amplifier [1], designed and being built by Omega-P, with a design power of over $40 \mathrm{MW}$ in a $1 \mu \mathrm{sec}$ pulse. Preliminary calculations indicate that this should make it possible to test the structure to accelerating gradients of over $300 \mathrm{MV} / \mathrm{m}$.
\end{abstract}

\section{INTRODUCTION}

Strong motivation for developing high-frequency accelerating structures follows from the scaling of accelerating gradient with frequency, which is approximately linear [2]. Thus ILC, which is to operate at $11.424 \mathrm{GHz}$, is to achieve an unloaded accelerating gradient of about $77 \mathrm{MeV} / \mathrm{m}$ [3], approximately four times greater than the $2.856 \mathrm{GHz}$ SLC. Similarily, at $34 \mathrm{GHz}$ the acceleration gradient could exceed $200 \mathrm{MeV} / \mathrm{m}$. Laboratory tests would allow confirmation (or not) of the widely accepted scaling with frequency of field emission and $\mathrm{rf}$ breakdown in high-gradient room-temperature linac structures [4]. Tests has been performed with microsecond-length pulses at S, C and X-band; and at 30 $\mathrm{GHz}$ at relatively low power level with $12 \mathrm{nsec}$ pulses on the CLIC structure [5]. But extension upwards in power and pulse length are required to further test the scaling laws at $34.272 \mathrm{GHz}$ in a regime where conventional highgradient linacs would operate, namely with RF surface electric field gradients greater than $0.5 \mathrm{GeV} / \mathrm{m}$ and pulse widths greater than $100 \mathrm{nsec}$. With a $45 \mathrm{MW}$ magnicon amplifier at $34.272 \mathrm{GHz}$ under development by Omega-P, is it possible to consider such high-power tests of accelerating structures.

Making use of the favorable scaling of accelerating gradient with frequency assumes that RF source and pulse compressor technology will advance sufficiently so as to furnish of the order of $615 \mathrm{MW} / \mathrm{m}$ of required RF drive power to achieve $250 \mathrm{MeV} / \mathrm{m}$ accelerating gradient [2]. To push the structure up to $250 \mathrm{MeV} / \mathrm{m}$, which corresponds a surface field of $\sim 500 \mathrm{MV} / \mathrm{m}$ even for the 6- $\mathrm{cm}$ long structure described below, would require a peak RF power level of $120 \mathrm{MW}$. No $34.272 \mathrm{GHz}$ RF source with this power is available. Nor are power enhancement schemes, such as resonant rings or pulse compressors, available for this frequency and power level. Until such RF power enhancement technology becomes available, another approach is required to achieve the surface electric field of more than $500 \mathrm{MV} / \mathrm{m}$ in a $34.272 \mathrm{GHz}$ structure. This method is to operate a traveling-wave structure in a standing-wave mode, which can be arranged by placing an RF short circuit at the end of the structure under test. Under this condition, analysis of the energy spectrum of resulting dark current is a common diagnostic used to ascertain the acceleration gradient. Knowledge of the overvoltage factor then allows the maximum surface gradient to be inferred from the acceleration gradient.

\section{THE TEST STRUCTURE}

Parameters of the accelerating structure are to be chosen so as to achieve an accelerating gradient greater than 250 $\mathrm{MeV} / \mathrm{m}$, assuming the necessary RF peak power to be available. Although the design value of peak output power of the Omega-P magnicon is $45 \mathrm{MW}$ in a $1 \mu \mathrm{sec}$ pulse [1], a more conservative level of $30 \mathrm{MW}$ is chosen for the proposed project.

The customary accelerating structure for linear colliders is a $2 \pi / 3$ structure with a normalized group velocity $v_{g_{r}} / c$ in the range $0.03-0.1$. A schematic crossection of the structure is shown in the Fig. 1a. As was discussed in ref.

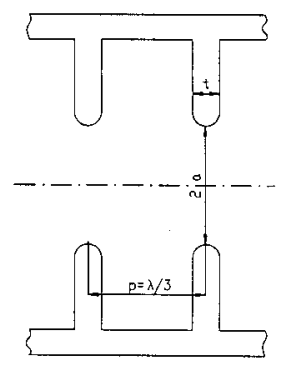

(a)

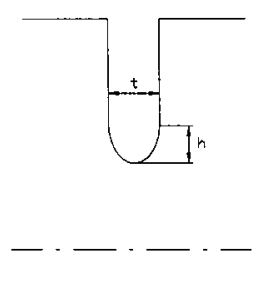

(b)
Fig. 1. A schematic cross section of the $2 \pi / 3$ structure (a), the disk edge geometry (b).

[4], the maximum accelerating gradient $E$ is determined mainly by the maximum electric field on the cavity surface $E_{m}$. Thus, the maximum accelerating gradient for fixed surface field is achieved, if the overvoltage factor 
$k_{m}=E_{m} / E$ is minimized. To minimize $k_{m}$, a disk edge geometry was suggested [6] having an elliptical shape, as shown in Fig. 1b. Numerical simulations show, within the range of group velocity $0.01 \leq v_{g_{r}} / c \leq 0.1$, and for the cavity wall thickness $t$ in the range of $0.056 \lambda$ to $0.11 \lambda$, a reduction in $k_{m}$ in the range of $5 \%$ to $40 \%$. Here $\lambda$ is the free-space wavelength at the design frequency. The next important issue is the choice of structure length, equivalent to a choice of the number of cells, to investigate effects which may not appear in a single cell. Naturally, the maximum number of cells is limited by the available peak RF power. For the discussion that follows, a structure having 21 cells was chosen. Table I lists results for some parameters of a preliminary design optimization for this structure.

Table I. Preliminary parameters for high-gradient 34 $\mathrm{GHz}$ accelerator structure.

\begin{tabular}{|l|l|}
\hline Operating frequency & $34.272 \mathrm{GHz}$ \\
Operating mode & $2 \pi / 3$ \\
Number of cells & 21 \\
Structure length & $62 \mathrm{~mm}$ \\
Group velocity & $0.048 \mathrm{c}$ \\
Diameter of cell & $7.35 \mathrm{~mm}$ \\
Diameter of aperture & $3.0 \mathrm{~mm}$ \\
Wall thickness $t$ & $0.70 \mathrm{~mm}$ \\
Eccentricity $t / 2 h$ & 0.70 \\
Overvoltage factor $k_{m}$ & 2.0 \\
Shunt impedance & $130 \mathrm{MOhm} / \mathrm{m}$ \\
Quality factor & 3,800 \\
\hline
\end{tabular}

The wall thickness value $t$ that was chosen takes into account mechanical strength and the need for radial cooling. The shape of the disc edge is chosen to minimize $k_{m}$ [6]. Note that $k_{m}$ for a round shape disk edge is 2.2, i.e. $10 \%$ larger than that found in the preliminary optimization of this structure.

The proposed structure and a corresponding field map in a standing wave mode are shown in Fig. 2. The diameter of the end cell is reduced to $7.26 \mathrm{~mm}$, as compared to 7.35 $\mathrm{mm}$ for the intermediate cells in order to compensate effect of a beam tunnel. The dispersion curve for this structure is shown in Fig. 3. These results were found using calculations with the code SLANS [7]. A $30 \mathrm{MW}$ peak power magnicon will allow to test this structure up to

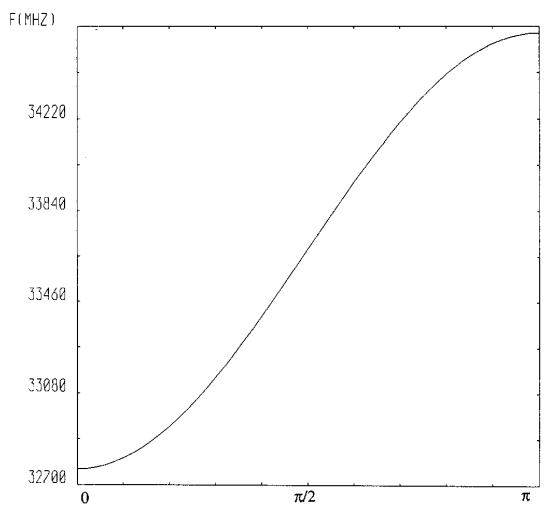

Fig. 3. Dispersion curve for the structure shown in Fig. 2.

maximum surface field of about $700 \mathrm{MV} / \mathrm{m}$. It should be emphasized that, in the case of standing-wave structures, the overvoltage factor $k_{m}$ is almost twice as high than for the traveling-wave case; in the present instance, $k_{m}=3.85$.

\section{THE INPUT COUPLERS}

Input couplers constitute another important issue. The approach which is taken for the $11.424 \mathrm{GHz}$ structure for NLC [8], and for the $30 \mathrm{GHz}$ structure for CLIC [5], is to use two symmetric input couplers. It has been shown recently [9] that quadrupolar field asymmetries, introduced by input irises having an azimuthal variation, will enhance the surface electric field in the input cell. This enhancement factor in some cases may exceed $25 \%$. To avoid this problem, a so called "racetrack" geometry for the input cavity was introduced [9]. Racetrack geometry allows one to symmetrize the fields; it has been shown to work well at $11.4 \mathrm{GHz}$ and $17 \mathrm{GHz}$. But at $34.272 \mathrm{GHz}$, additional problems arise due to the extremely high gradients and microwave power levels in the small size input waveguides. To feed the $34.272 \mathrm{GHz}$ structure described here, four symmetric input couplers will be used. This approach provides the following benefits:

- $\quad$ an increase in the order of perturbation asymmetry from quadrupole to octupole, with a concomitant decrease in the field enhancement factor to below $3 \%$;

- a factor-of-two decrease in power flow through each input waveguide; and

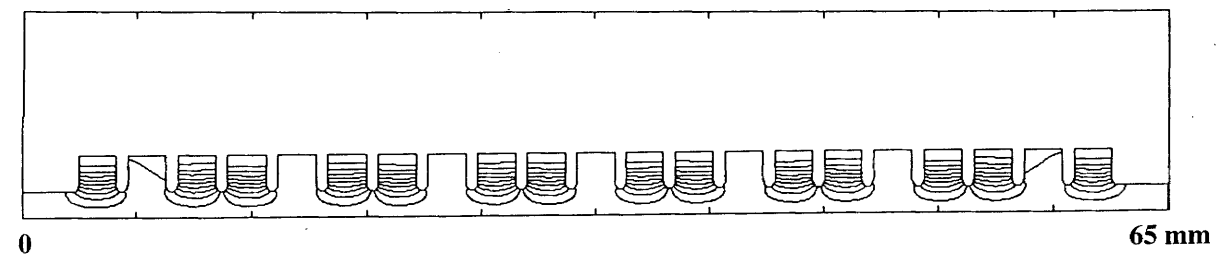

Fig. 2. Field map of $2 \pi / 3$ standing wave mode in the 21 -cell structure. 
- a straightforward connection between the magnicon [1] and the accelerator structure input coupler, since the magnicon has four WR-28 output waveguides; thus avoiding need for a complex power divider for input power distribution.

A field map of the input cell with four WR-28 waveguides is shown in Fig. 4. 2-D simulations were carried out for the case of perfect matching, using CLANS code [7]. Width of the coupling irises corresponds to the case of standing-wave operation of the structure. Zoomed picture of this field map is shown in the Fig. 5. One can see that near the structure axis magnetic force lines are practically circular, indicating that only a small field enhancement factor is present.

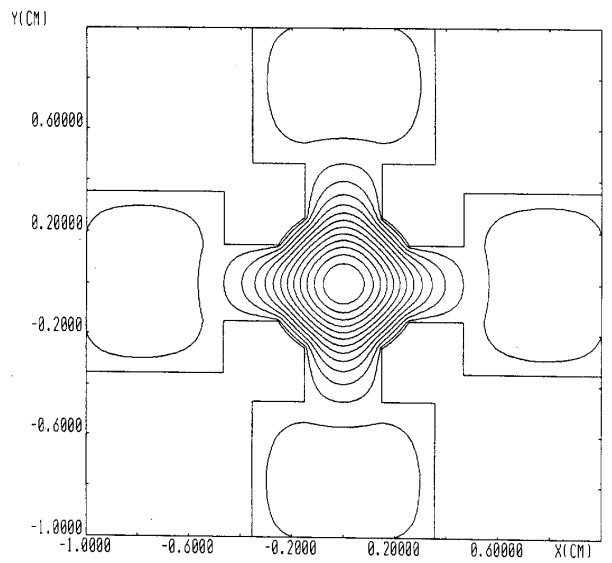

Fig. 4. Field map in the transverse cross section of the input cell.

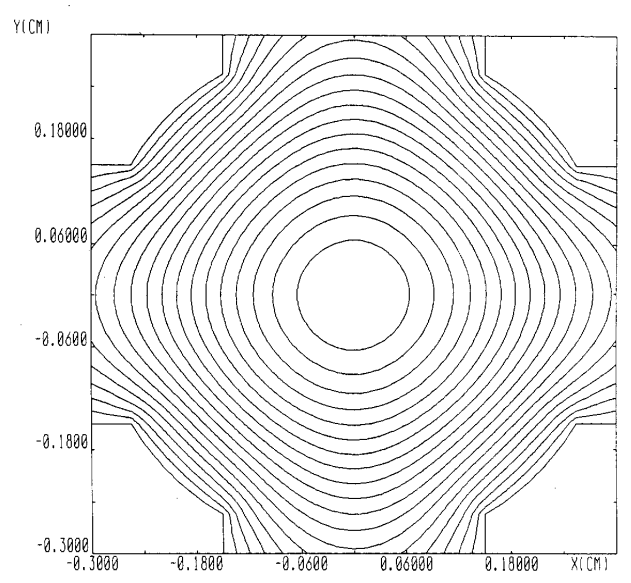

Fig. 5. Zoomed field map, showing good axial symmetry in the center of the input cell.

\section{CONCLUSIONS}

Continued progress in high energy physics demands a new generation of linear collider, which in turn requires operation at frequencies higher than $11.4 \mathrm{GHz}$, the frequency chosen for NLC. At $34 \mathrm{GHz}$, it is expected that an acceleration gradient approximately three times that at $11.4 \mathrm{GHz}$ can be realized. High-power tests on candidate structure are necessary to confirm this hypothesis. These tests on trial accelerator structure at $34 \mathrm{GHz}$ will be possible since a $45 \mathrm{MW}, 34 \mathrm{GHz}$ magnicon is being built by Omega-P, Inc. In particular, the tests of this accelerator structure may allow a surface gradient of $700 \mathrm{MV} / \mathrm{m}$, which corresponds to an accelerating gradient of more that $300 \mathrm{MeV} / \mathrm{m}$ for a travelling-wave accelerating structure.

\section{ACKNOWLEDGMENTS}

This research was supported by the US Department of Energy, Division of High Energy Physics. The authors would like to thank Dr. J. L. Hirshfield for careful reading of the manuscript.

\section{REFERENCES}

[1] O. A. Nezhevenko, V. P. Yakovlev, A. K. Ganguly, J. L. Hirshfield, "High Power Pulsed Magnicon at $34 \mathrm{GHz}$ ", $R F$ '98 Workshop, Pajaro Dunes, CA, October 5-9, 1998 (to be published)

[2] P. B. Wilson, "Scaling Linear Colliders to $5 \mathrm{TeV}$ and Above", SLAC-PUB-7449, April, 1997.

[3] T. O. Raubenheimer, K Yokoya, "Proposed ILC Parameters", SLAC Note 06-05-98.

[4] J. W. Wang, G. A. Loew, "Field Emission and RF Breakdown in High-Gradient - Room-Temperature Structures", SLAC-PUB-7684, October 1997

[5] H. H. Braun, K. Aulenbacher, R. Bosart, F. Cautard, R. Corsini, J. P. Delahaye, J. C. Godot, S. Hutchins, I. Kamber, J.H.B. Madsen, L. Rinolfi, G. Rossat, S. Schreiberg, G. Suberlucq, L. Thorndahl, I. Wilson, W. Wuensch, "Results From the CLIC Test Facility", Proc. 1996 European Particle Accel. Conf., , pp. 42-46.

[6] O. Nezhevenko, D. Myakishev, V. Tarnetsky, V. Yakovlev, "TW Accelerating Structures with Minimal Electric Field", Proc. 1995 Particle Accelerator Conference and International Conference on High Energy Accelerators, Dallas, 1995, pp. 1076- 1078.

[7] D. Myakishev, V. Yakovlev, "The New Possibilities of SuperLANS Code", Proc. of 1995 Particle Accelerator Conference and International Conference on High Energy Accelerators, Dallas, 1995, pp.2348-2350.

[8] K.A. Tomson et al, "Design and Simulation of Accelerating Structures for Future Linear Colliders", in Particle Accelerators, 1994, vol.47, pp. 65-109.

[9] J. Haimson, B. Mecklenburg and E.L. Wright, "A Racetrack Geometry to Avoid Undesirable Azimuthal Variations of the Electric Field Gradient in High Power Coupling Cavities for TW Structures", Proc. of $7^{\text {th }}$ AAC Workshop, Lake Tahoe, October 1996, pp. 898-911. 